\title{
CD206+ M2-Like Macrophages Are Essential for Successful Implantation
}

\begin{abstract}
Yosuke Ono ${ }^{1}$, Osamu Yoshino ${ }^{2 *}$, Takehiro Hiraoka ${ }^{2}$, Erina Sato ${ }^{2}$, Yamato Fukui ${ }^{3}$, Akemi Ushijima ${ }^{4}$, Allah Nawaz ${ }^{5}$, Yasushi Hirota ${ }^{3}$, Shinichiro Wada ${ }^{1}$, Kazuyuki Tobe ${ }^{6}$, Akitoshi Nakashima ${ }^{4}$, Yutaka Osuga ${ }^{3}$ and Shigeru Saito ${ }^{4 *}$

${ }^{1}$ Department of Obstetrics and Gynecology, Teine Keijinkai Hospital, Sapporo, Japan, ${ }^{2}$ Department of Obstetrics and Gynecology, Kitasato University School Medicine, Tokyo, Japan, ${ }^{3}$ Department of Obstetrics and Gynecology, Faculty of Medicine, University of Tokyo, Tokyo, Japan, ${ }^{4}$ Department of Obstetrics and Gynecology, Faculty of Medicine, University of Toyama, Toyama, Japan, ${ }^{5}$ Department of Molecular and Medical Pharmacology, Faculty of Medicine, University of Toyama, Toyama, Japan, ${ }^{6}$ First Department of Internal Medicine, University of Toyama, Toyama, Japan
\end{abstract}

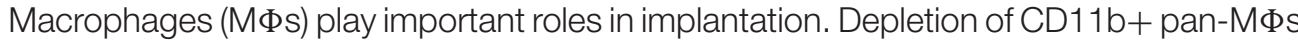
in CD11b-diphtheria-toxin-receptor (DTR) mice is reported to cause implantation failure due to decreased progesterone production in the corpus luteum. However, of the M1 and $\mathrm{M} 2$, the type of MФs that is important for implantation is unknown. In this study, we investigated the role of M2 M $\Phi$ in implantation using CD206-DTR mice. To deplete M2-MФ, female CD206-DTR C57/BL6 mice were injected with DT before implantation. These M2-M $\Phi$ depleted mice (M2(-)) were naturally mated with Balb/C mice. As the control group, female C57/BL6 wild type (WT) mice injected with DT were mated with male Balb/C mice. The number of implantation sites and plasma progesterone levels at implantation were examined. Implantation-related molecule expression was determined using quantitative-PCR and immunohistochemistry of uterine tissues. The mRNA expression in the endometrial tissues of 38 patients with implantation failure was examined during the implantation window. In WT mice, CD206+M2-like MФs accumulated in the endometrium at the implantation period, on embryonic (E) 4.5. In M2(-), the implantation number was significantly lower than that in control $(p<0.001$, $7.8 \pm 0.8$ vs. $0.2 \pm 0.4$ ), although the plasma progesterone levels were not changed. Leukemia inhibitory factor (LIF) and CD206 mRNA expression was significantly reduced $(p<0.01)$, whereas the levels of TNF $\alpha$ were increased on E4.5 $(p<0.05)$. In M2(-), the number of Ki-67+ epithelial cells was higher than that in control at the pre-implantation period. Accelerated epithelial cell proliferation was confirmed by significantly upregulated uterine fibroblast growth factor (FGF)18 mRNA ( $P<0.05$ ), and strong FGF18 protein expression in M2(-) endometrial epithelial cells. Further, M2(-) showed upregulated uterine Wnt/ $\beta$-catenin signals at the mRNA and protein levels. In the non-pregnant group, the proportion of M2-like M $\Phi$ to pan M $\Phi$, CD206/CD68, was significantly reduced $(p<0.05)$ and the TNF $\alpha$ mRNA expression was significantly increased $(p<0.05)$ in the endometrial tissues compared to those in the pregnant group. CD206+ M2-like MФs may be essential for embryo implantation through the regulation of endometrial proliferation via Wnt/ $\beta$-catenin signaling.

Keywords: CD206, diphtheria-toxin receptor mouse, fibroblast growth factor, implantation, M2 macrophage, Wnt/ $\beta$-catenin signal 


\section{INTRODUCTION}

Macrophages $(M \Phi)$ are a crucial player in the generation and execution of immune responses through various functions, including phagocytosis, antigen presentation, and secretion of a variety of cytokines and growth factors (1-3). Recently, $M \Phi$ s have been reported to play an essential role in tissue development and homeostasis through increased angiogenesis and vascular remodeling $(1,4,5) . M \Phi s$ also have attracted significant interest in human diseases as they play crucial roles in many diseases associated with chronic inflammation such as atherosclerosis, obesity, diabetes, cancer, skin diseases, and neurodegenerative diseases $(6,7)$. Implantation is a vital process of the first feto-maternal encounter in the uterus, leading to pregnancy. Good coordination between a blastocyst and receptive uterus is essential for successful implantation $(8,9)$. Although implantation is an important phenomenon in pregnancy, its precise mechanism is not fully understood due to its complexity involving multi-factors. Animal studies using different kinds of genetically altered mice have been undertaken to elucidate the mechanism of implantation (10-13). Although few studies have examined the relationship between $M \Phi$ and implantation, Care et al. first reported that $M \Phi$ plays an important role in the implantation process in CD11b-DTR mice (14). They showed that depletion of CD11b $+M \Phi$ s resulted in the implantation failure due to decreased progesterone production in the corpus luteum (14). MФs are classified into two subtypes,

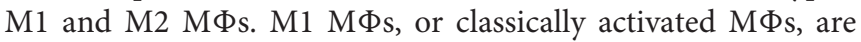
pro-inflammatory and play a central role in host defense against

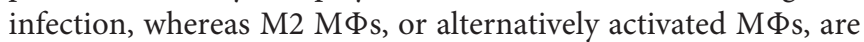
associated with responses to anti-inflammatory reactions and tissue remodeling (15).

The precise role of $\mathrm{M} \Phi$ s in the uterus at the implantation period is unclear in implantation period.

$\mathrm{M} \Phi$ s demonstrate plasticity and polarize to the M1 or M2 type according to their surrounding microenvironment and stimuli $(2,16)$ and skewness to M1 or M2 MФs has been reported in various diseases (4). But it is not clear which type of MФs mostly contributes to the implantation. In the present study, we investigated the role of CD206+ M2-like MФ in implantation using CD206-diphtheria-toxin (DT)-receptor transgenic mice (17-19), in which M2-like MФs can be specifically depleted.

\section{RESULTS}

\section{CD206+M2-Like Mकs Are Located in the Uterus at the Implantation Period}

Most $M \Phi s$ in non-pregnant mice are known to be present in the uterine stroma, but are reported to exist in the lumen and glands during the implantation period (20). To examine the localization of M2-like M $\Phi$ s in the uterus at the implantation period on embryonic day 4.5 (E4.5), we performed CD206 immunohistochemistry in wild type (WT) mice. At the implantation period, we found that CD206+ M2-like MФs were located in the uterine stromal region as well as close to the lumen and glands. Immunofluorescence analysis revealed that CD206+ cells were found in WT with DT and TG with PBS group, while these were completely depleted in TG with DT mice (Figure 1Aa). To examine the change of M2-like $M \Phi s$ in the uterus at the implantation period, we compared CD206 mRNA expressions between non-pregnancy and implantation periods. The mRNA expressions of uterine CD206 was significantly increased during implantation period, peaking at embryonic (E) 3.5, compared to non-pregnancy (Figure 1A-b).

\section{Implantation Was Impaired in the M2(-) Group}

To investigate the role of CD206+ M2-like MФ in implantation, we set the protocol of an implantation model using CD206 DTR mice (Figure 1B). We naturally mated C57/B6 female mice with Balb/c male mice as controls, or mated CD206 female DTR mice with Balb/c mice, defined as M2(-). DT was intraperitoneally administered to the control and M2(-) mice before the implantation period. The depletion of CD206+ M2like $\mathrm{M} \Phi$ s was checked by $\mathrm{qPCR}$. We compared the number of implantation sites between the two groups at E4.5 after in Chicago Blue dye administration. The implantation sites in M2(-) mice were significantly fewer compared to those in the control mice $(P<0.001,7.8 \pm 0.8$ vs. $0.2 \pm 0.4)$ (Figures 2A,B). As leukemia inhibitory factor (LIF)-Stat signaling is known to be essential for implantation (21), LIF mRNA expression was examined in the uterine tissues in M2(-) mice and was found to be significantly decreased compared to that in control $(p<0.05$, Figure 3A). Immunostaining for phosphorylated Stat 3 was also found in uterine epithelial cells of control mice but not in M2(-) mice (Figure 3B). The proportion of phosphorilated (p) STAT3positive epithelial/total epithelial cells was significantly reduced in the M2(-) group compared to control (mean $\pm \mathrm{SD}, 38.5 \pm$ $13.2 \%$ vs. $0 \%$; $p<0.01$, Figure $3 \mathrm{C}$ ).

\section{The Accelerated Proliferation of Epithelial Cells Was Found in M2(-) Mice}

We examined the morphological changes in the just after implantation period (E 5.5). In M2(-) mice, cell proliferation in the stromal region was impaired, and epithelial cells were proliferative compared to control (Figure 4A). We then examined the cell proliferation at pre-implantation period (E3.5). In M2(-) mice, the number of Ki-67-positive epithelial cells was higher compared to that in control at the pre-implantation period (E3.5). However, there were no histological differences in the corpus luteum and the plasma $\mathrm{P} 4$ concentration between both groups (Figures $4 \mathrm{~B}, \mathrm{C}$ ). These suggest that endometrial epithelial cells had not transformed to become receptive to embryo implantation (Figure 5).

\section{Uterine Wnt/ $\beta$-Catenin Signaling Is Upregulated in M2(-) Mice at the Pre-implantation Period (E3.5)}

Uterine $W n t / \beta$-catenin signals regulate the production of fibroblast growth factor (FGF), and proper modification of these signals is essential for implantation (22). In M2(-) mice, at the implantation period, the mRNA expression of Wnt 4A, Wnt 7B, and $\beta$-catenin, was significantly increased $(p<0.05)$ compared 


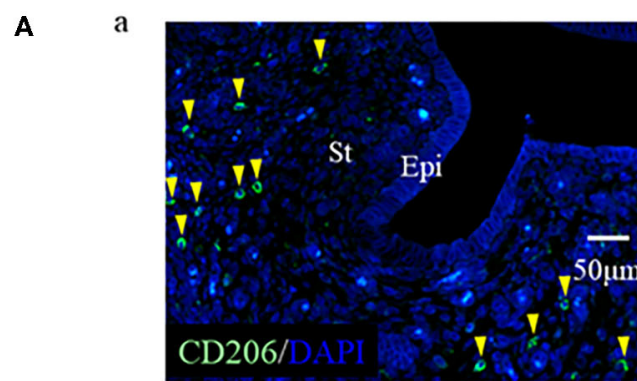

(i) WT+DT

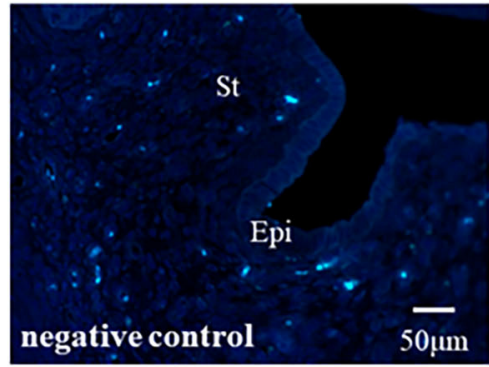

(iv) WT+DT

Epi: epithelium cells $\quad$ St; stroma cells

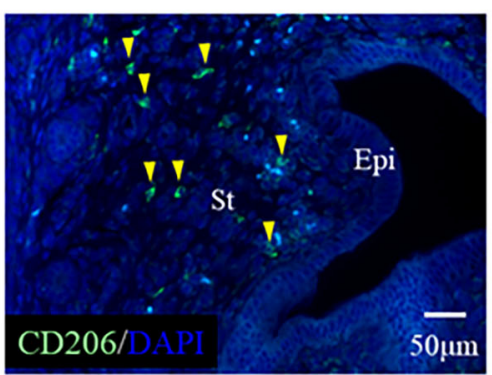

(ii) $\mathrm{TG}+\mathrm{PBS}$

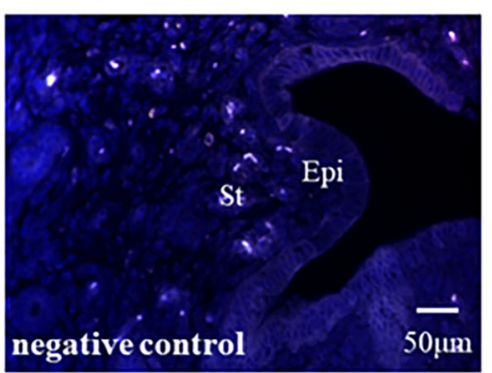

(v) $\mathrm{TG}+\mathrm{PBS}$

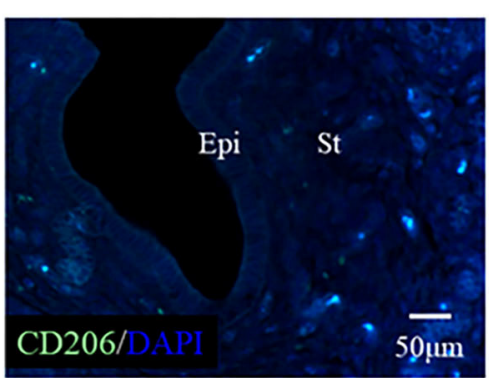

(iii) $\mathrm{TG}+\mathrm{DT}$

b

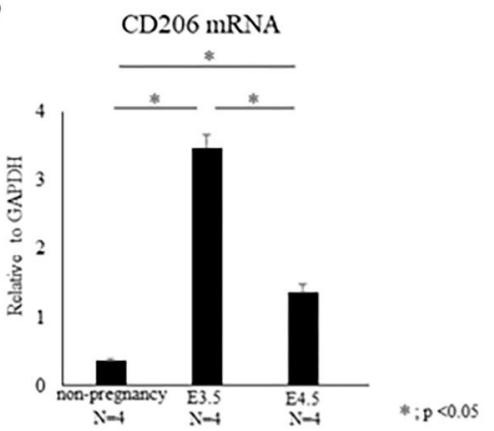

B

(1) control group 우 $\mathrm{C} 57 \mathrm{~B} 6 \times$ 우 $\mathrm{Balb} / \mathrm{c}$

(2) M2(-) group 우 CD206 DTR (C57B6) $\times$ 우 Balb/c

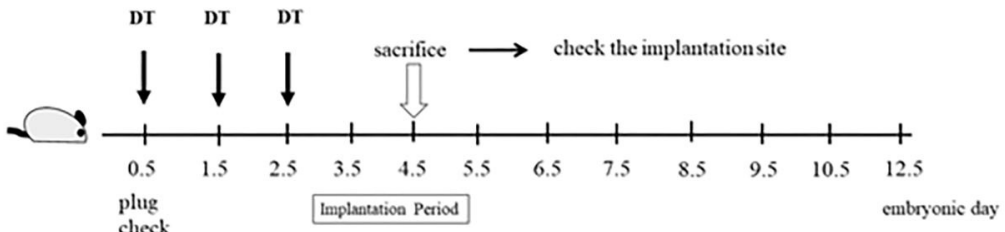

FIGURE 1 | (A) Localization of CD206+ M2-like macrophages (M $\Phi)$ in the uterus. Immunofluorescence for CD206 was performed for the uterus at embryonic day 4.5 (E4.5) in wild type (WT) and CD206 diphtheria toxin receptor transgenic mice (TG). The data of WT+DT(i), TG+PBS (ii), TG+DT (iii), WT+DT (iv), and TG+PBS(v) were shown. Anti- CD 206 antibody (i, ii, and iii) or control rabbit lgG (iv and v) were used for primary antibody. CD206-positive cells are in green, and nucleus were stained in blue. Yellow arrow heads show CD206+ M2-like MФ. The uterine mRNA expression of CD206 at non pregnancy, E3.5 and E4.5 are shown in (b). Data were normalized to GAPDH mRNA levels to determine the relative abundance and are shown as the mean \pm SEM. ${ }^{*} p<0.05$. (B) Depletion protocol for CD206+ M2-like macrophages (MФs) in the implantation model using CD206 DTR mouse. After checking the plug, diphtheria-toxin (DT) was intra-peritoneally injected prior to the implantation period (embryonic day; E0.5, E1.5, E2.5) in CD206-DTR female mice naturally mated with Balb/C male mice, defined as the M2(-) group. DT was also injected to C57/B6BL wild type (WT) female mice mated with Balb/C male mice, and were defined as the control group. The number of implantation sites and plasma progesterone levels at the implantation period (E4.5) were examined.

to the control, and endometrial epithelial cells exhibited strong staining for active $\beta$-catenin (Figure 6A). In detail, the basal site of uterine epithelial cells was strongly stained with $\beta$ catenin in M2(-) mice (Figure 6B). As expected, the mRNA expression of FGF18, downstream of the $\mathrm{Wnt} / \beta$-catenin signal, was significantly upregulated $(P<0.05)$ compared to that in control; further, FGF18 protein was also strongly stained in the endometrial epithelial cells of M2(-) mice (Figure 6C).

\section{Uterine Wnt/ $\beta$-Catenin Signaling Was Enhanced by Inflammatory M1-Like M $\Phi$}

Wnt signaling is reported to be increased by $\mathrm{TNF} \alpha$ in gastric tumor cells (23). We also found upregulated expression of TNF $\alpha$,
iNOS, and CD11c mRNAs produced by M1-like MФs (4) in the uterus of M2(-) mice compared to control $(p<0.05)$ (Figure 7).

\section{The Proportion of CD206+ M2-Like M\$s Among Total M $\Phi s$ in Uterine Tissues Was Significantly Reduced in Patients With Infertility}

We performed uterine endometrial biopsy in cohort of 38 infertility patients at the time of the implantation window. Implantation failure was diagnosed as the infertility factor for all these patients. After the endometrial biopsy, 19 patients got pregnant with assisted reproductive technology. The median age of the non-pregnant and the pregnant group was 40 


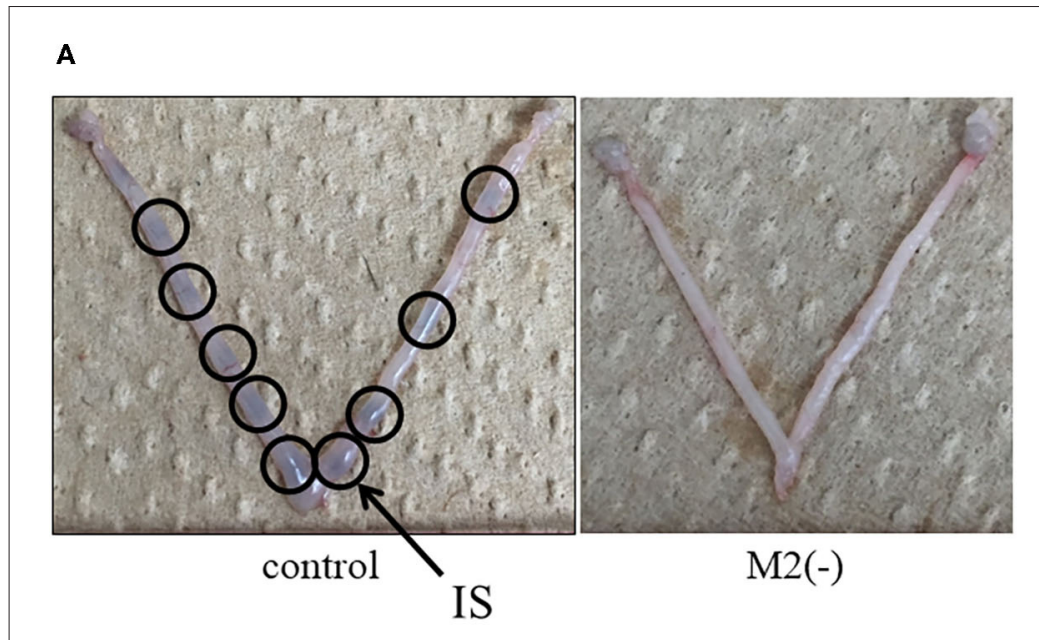

B

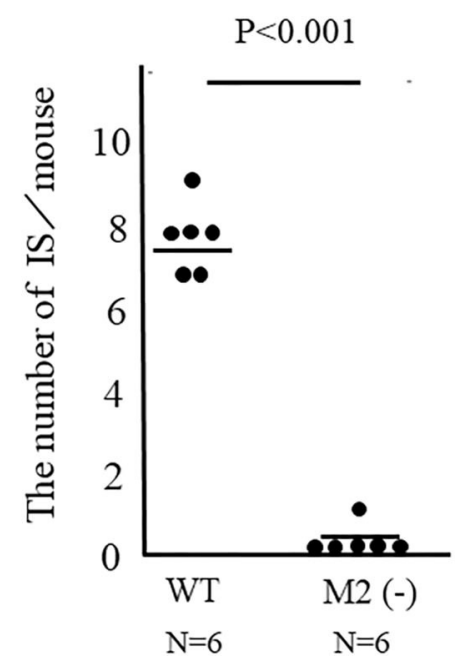

FIGURE 2 | Assessment of implantation site (IS). Representative appearances of the IS in CD206 DTR with DT (M2(-)) and control. ISs were stained with Chicago blue dye for facilitating their detection. ISs are shown (arrows) (A) and the number of ISs are shown as dots and the mean (B).

A

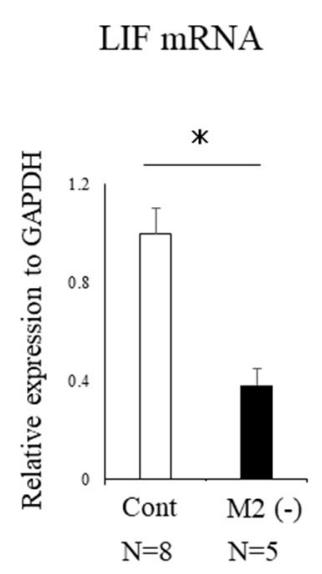

B

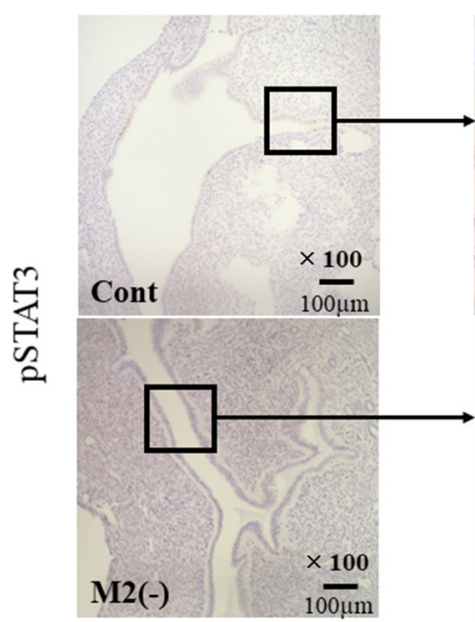

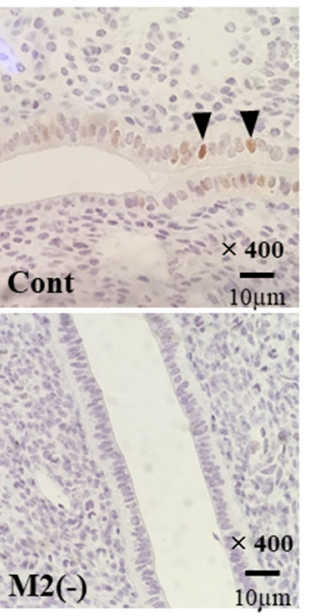

C

The proportion of pSTAT3-positive epithelial cells/ total epithelial cells

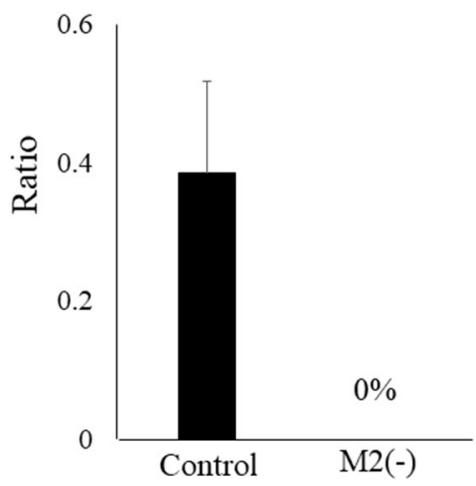

FIGURE 3 | LIF-STAT signal in the uterus at the implantation period. LIF mRNA expression in the uterus was examined. Data were normalized to GAPDH mRNA levels to determine the relative abundance and shown as (A). ${ }^{*} p<0.05$. Immunohistochemistry was performed for phosphorylated (p) STAT3 in the control and M2(-) mice (B). We randomly selected six different sites on the section of immunostaining and counted the number of phosphorylation (p) STAT3-positive epithelial cells, which were divided by the total number of epithelial cells between both groups. The proportion of pSTAT3-positive epithelial cells/ total epithelial cells was examined (C).

(29-44) years and 37.5 (33-43) years old, respectively, which was comparable between two groups. We then compared the

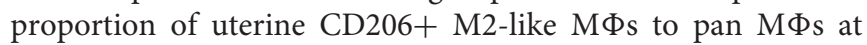
the mRNA levels of CD206/CD68, between pregnancy and nonpregnant groups. The relative ratio of $\mathrm{M} 2$-like $\mathrm{M} \Phi$ to total $\mathrm{M} \Phi$ was significantly reduced $(P<0.05)$ in non-pregnant group compared to that in the pregnant group, while upregulation of TNF $\alpha$ mRNA expression was observed in the non-pregnant group $(p<0.05)$ (Figure 8).

\section{DISCUSSION}

This is the first report investigating the role of M2 MФs in the uterus at the implantation period in the mouse model.
Previous reports showed that $\mathrm{M} \Phi$ s are important regulator of implantation and their depletion disrupts luteal vasculature resulting in reduced progesterone production from corpus luteum, which cause implantation failure $(14,24)$. These data suggest that depletion of pan $M \Phi$ s during the implantation period causes implantation failure not owing to defects in the uterus but attributed to the ovary. Plaks et al. used the CD11c DTR mouse model to report that uterine dendric cells (DCs) are essential for embryonic implantation (25). However, as CD11cpositive cells include both M $\Phi$ s and DCs, there was a limitation to determining the effect of each cell on implantation when CD11c cells were depleted. In the present study, we showed for the first time that CD206+ M2-like MФs are essential to implantation by using CD206 DTR mice. 


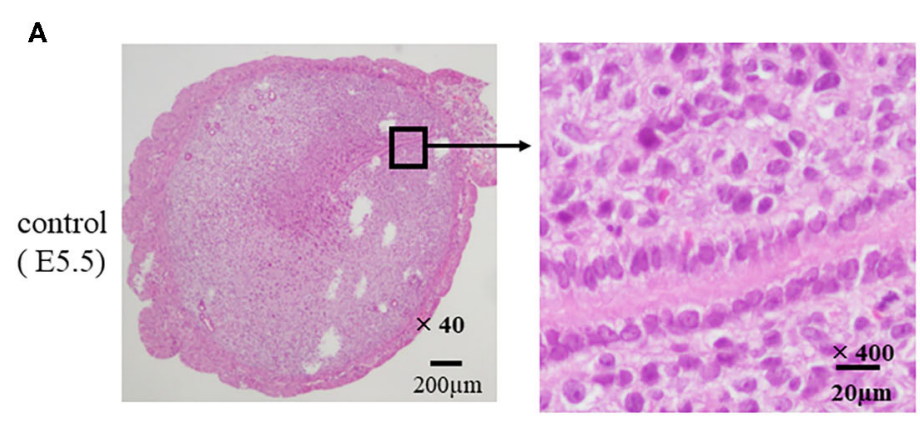

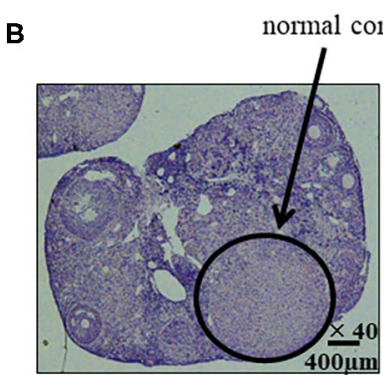

cont (E 3.5)

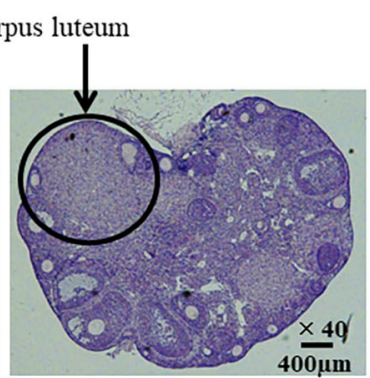

M2 (-) (E 3.5)

C

plasma progesterone (E 3.5)

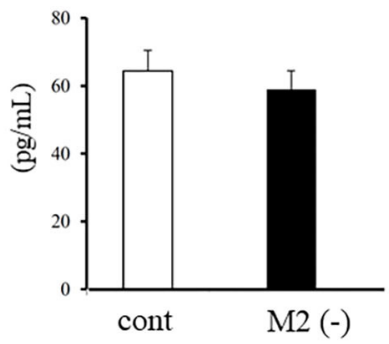

FIGURE 4 | Cross section of uterus and ovary. The cross sections of the implantation site (IS) are stained by hematoxylin and eosin (HE). The representative HE sections are shown at lower (x40) and higher (x400) magnification in the control and M2(-) at E5.5 (A). The corpus luteum [(B), hematoxylin and eosin stain] and the plasma progesterone levels $(\mathbf{C})$ in control and M2(-) at E 3.5 are shown.

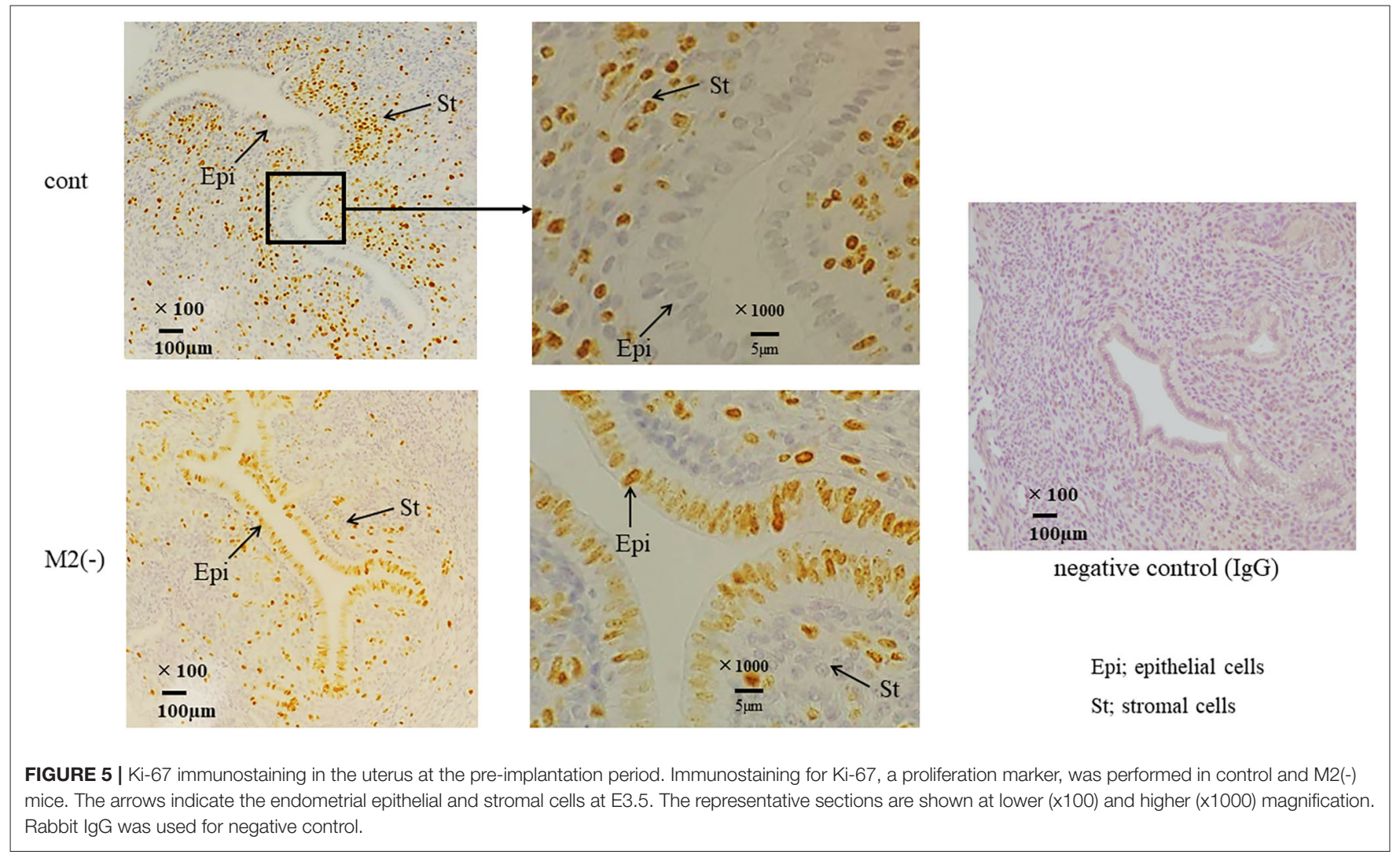



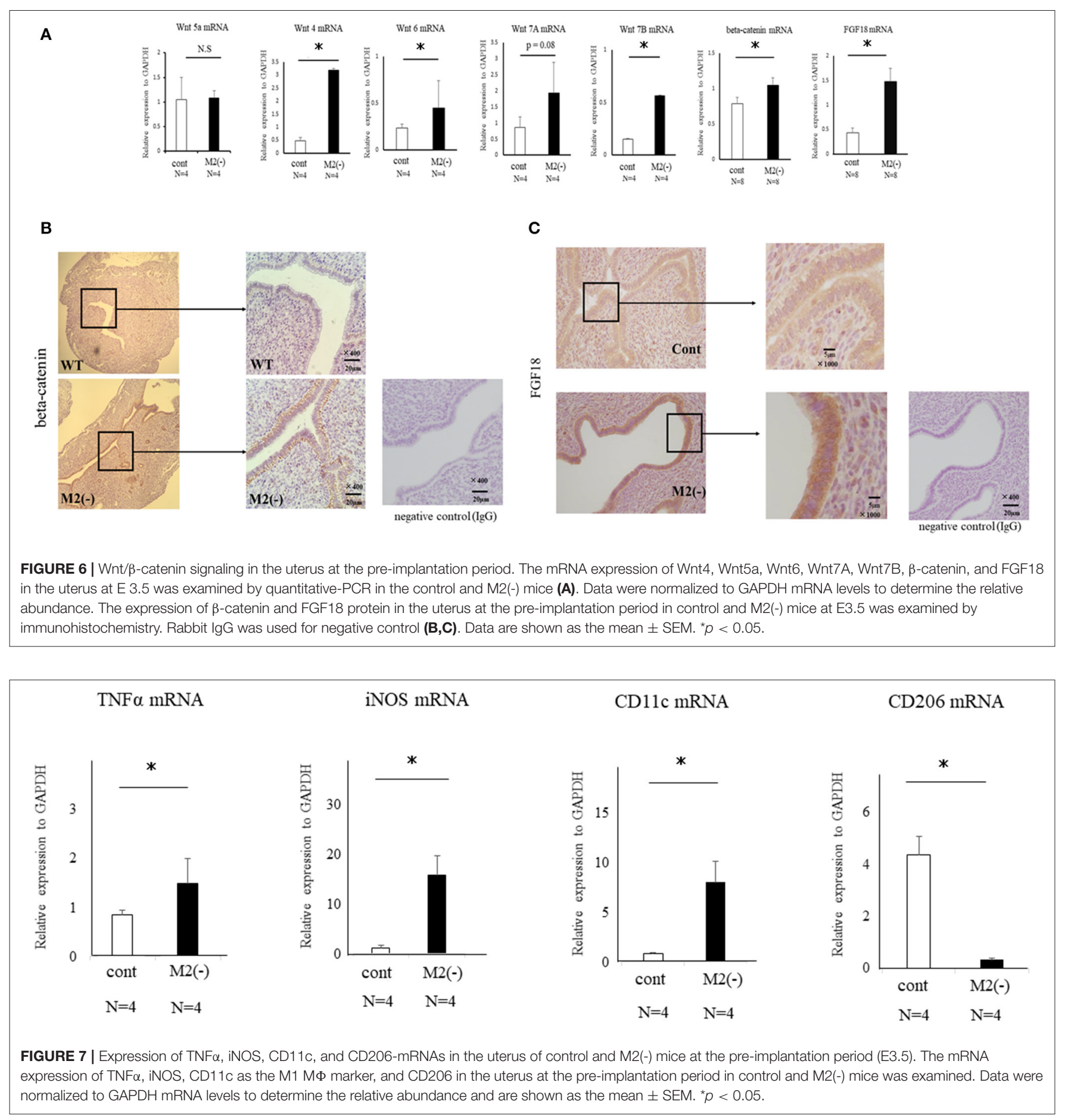

In the CD206 DTR mouse model, implantation failure occurred exclusively by the depletion of CD206+ M2-like MФs. As we have reported previously (19), we also found that the histological structure of the corpus luteum in M2(-) was not different from that of the control mice, and the plasma P4 levels were not changed, suggesting that the ovarian function at the implantation period was maintained in the absence of M2-like MФs. Reduced plasma progesterone level by luteal dysfunction in depletion of pan $M \Phi$ s mice model might be caused by the depletion of M1 МФs. Therefore, the implantation failure may be attributed to the abnormal interaction between the embryo and uterus. In our previous study, we examined the effects of oocytes and embryos quality derived from $\mathrm{M} 2 \mathrm{M} \Phi$ depletion mouse on fertilization and implantation (19). In detail, after inducing superovulation in wild type (WT) and CD206+M2-like M $\Phi$ depleted mice, oocytes obtained from the fallopian tubes 

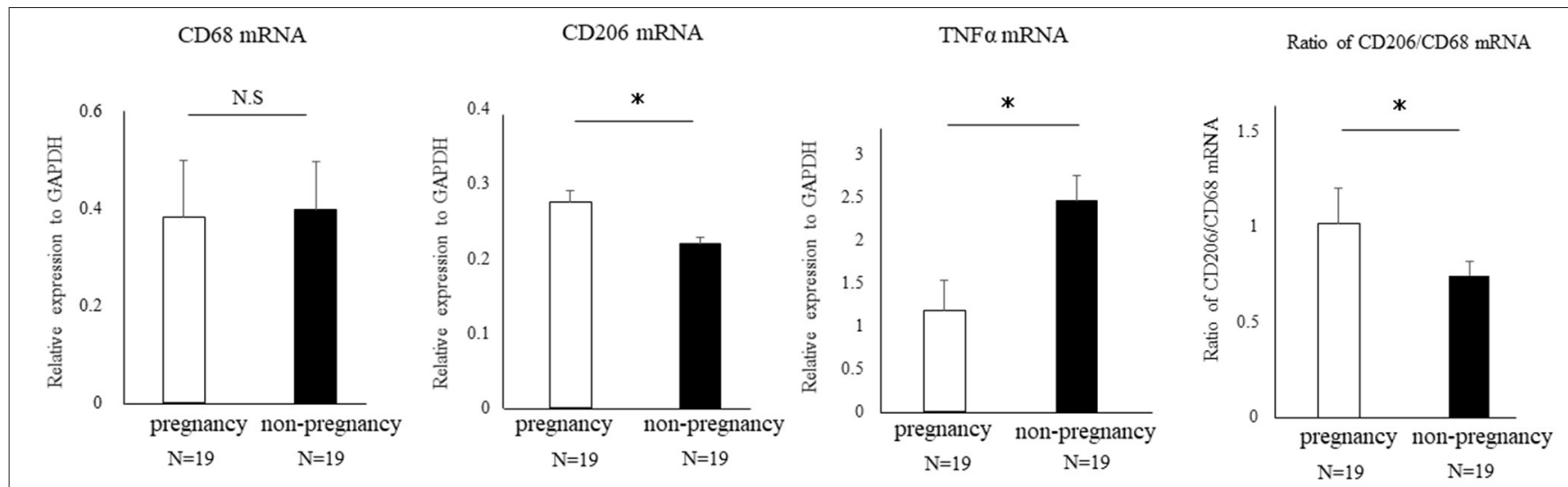

FIGURE 8 | The proportion of uterine CD206+ M2-like MФ in human cases of pregnancy success and failure. A cohort of 38 patients with implantation failure were subjected to uterine endometrial biopsy at the timing of the implantation window. The mRNA expression of CD68 (pan-M $\Phi$ ), CD206, and TNF $\alpha$ in uterus were examined between the pregnant and non-pregnant group after endometrial biopsy. Data were normalized to GAPDH mRNA levels to demonstrate the relative

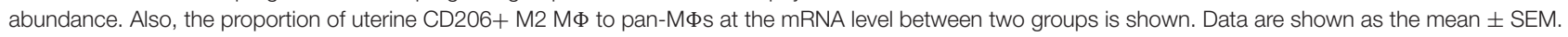
${ }^{\star} p<0.05$.

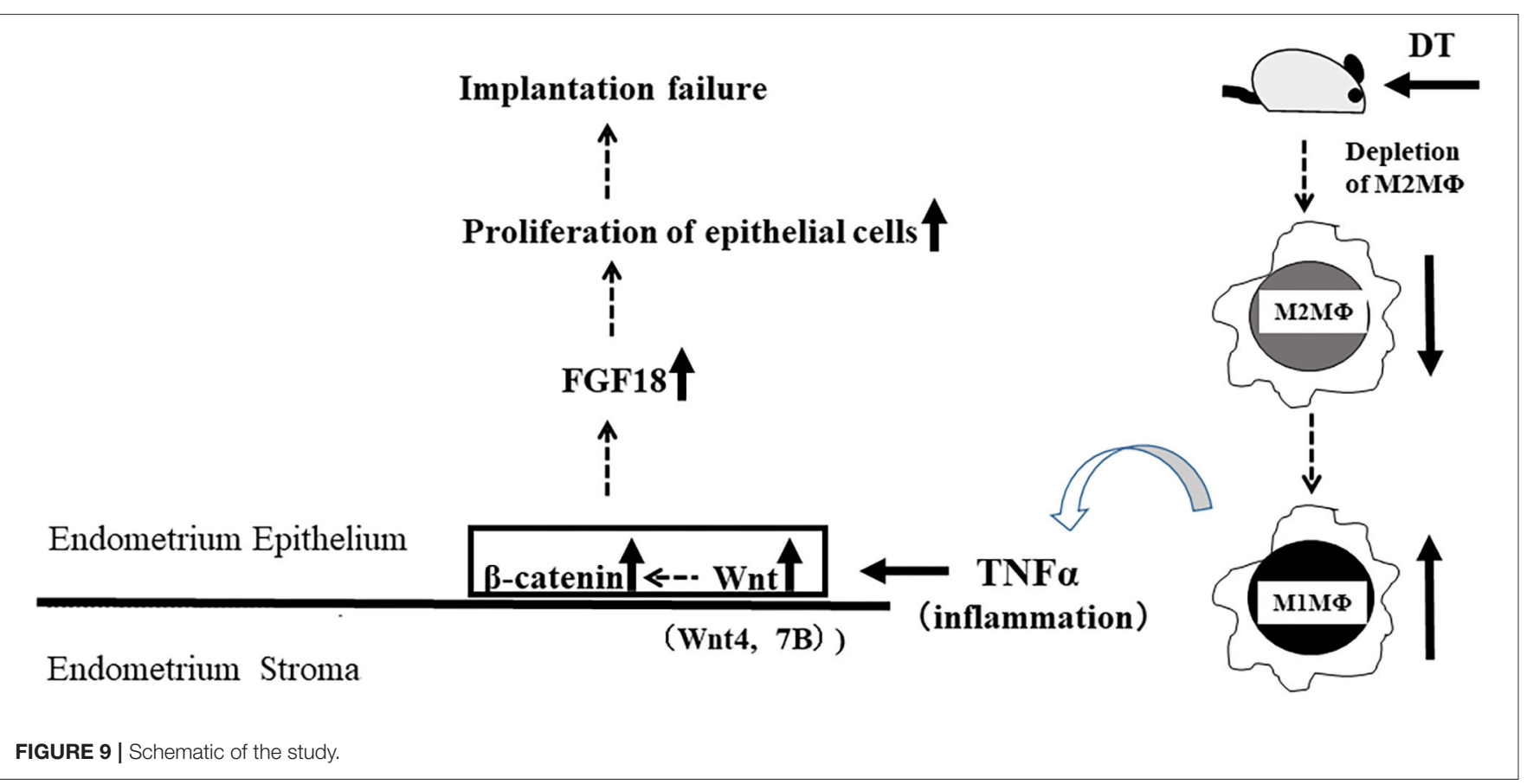

of these mice were in vitro fertilized, followed by transferring to pseudo pregnant WT mice. The fertilization rate, blastocyst formation rate, and pregnancy rate of CD206 DTR-mice derived oocytes were comparable to that of WT-mice derived oocytes, suggesting that oocytes derived from CD206+M2-like МФdepleted mice did not affect fertilization and implantation (19). In the present study, the structure of the corpus luteum and the plasma progesterone level was maintained during the implantation period. We detected morphological abnormality only in the uterus, so decreased uterine MФs were considered the cause of implantation failure. In addition, at the preimplantation period (E3.5), embryos obtained by flushing the uterine cavity with saline in both WT and CD206 DTR mice exhibited no morphological differences (data not shown). These data suggest that the depletion of $\mathrm{M} 2 \mathrm{M} \Phi$ in vivo did not affect the embryo quality and hormonal milieu. Therefore, the cause of implantation failure in M2(-) mice as observed in the present study, was the uterus and was not due to the abnormalities in the ovary or the embryo. Subsequently, we focused on the role of M2 MФs in the endometrium during implantation. Elevated P4 concentrations after ovulation dramatically change the state of endometrial cell proliferation and render the uterus receptive to the embryo as a normal uterine morphological change that occurs during pre-implantation (26). In normal conditions, 
luminal epithelial cells are known to cease proliferation for implantation $(26,27)$; however, in M2(-) mice, the number of Ki-67-positive endometrial epithelial cells was higher compared to the control at the preimplantation period (E3.5), suggesting that the endometrial epithelial cells did not undergo the required change for receiving the embryo (Figure 5).

Nallasamy et al. have reported that targeted mutation of the homeobox transcription factors, Msx1 and Msx2, which control organogenesis and tissue interactions during embryonic development, in both the uterine epithelium and stroma, results in implantation failure. Based on gene expression profiling of the uterine epithelium and stroma from Msx1/2d/d mice, elevation of $\mathrm{Wnt} / \beta$-catenin signaling leads to an increase in fibroblast growth factor (FGF) production in the uterine stroma (28). Moreover, upregulated FGFs act in a paracrine manner on the uterine epithelium to promote epithelial proliferation, which prevents endometrial differentiation and creates a non-receptive uterus for the embryo (28). This indicates that an excessive increase in $W n t / \beta$-catenin signaling leads to an unreceptive uterus, which is refractory to implantation due to its inability to control epithelial proliferation, though moderately balanced uterine Wnt/ $\beta$-catenin signaling is reported to be necessary for implantation (22). In the present study, the accelerated proliferation of epithelial cells in M2(-) mice might be due to a higher expression of FGF-18 in endometrial epithelial cells.

Aberrant expression of TNF $\alpha$ has been reported as one of the causes of enhanced $W n t / \beta$-catenin signaling (23). In the present M2(-) mouse model, we found that the expression of $\mathrm{TNF} \alpha$ and M1-like M $\Phi$ markers such as inducible nitric oxide synthase (iNOs) and CD11c (10), were significantly increased at the mRNA level $(P<0.05)$. This upregulation of M1-like $M \Phi$ related molecules might be due to a relative increase in M1-like MФs owing to the depletion of CD206+ M2-like MФs. Kambara et al. also reported that the expression of proinflammatory cytokines such as TNF $\alpha$, IL- $1 \beta$, IL-6, and MCP1 were significantly upregulated in the lung tissues of CD206 DTR mice in response to DT treatment (17). Collectively, we hypothesized that upregulation of TNF $\alpha$ secreted by M1-like

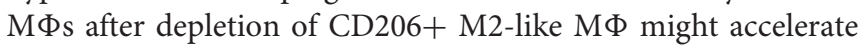
the uterine Wnt/ $\beta$-catenin signal in endometrial epithelial cells. Further, in epithelial cells, FGF18 expression was increased aberrantly, resulting in the proliferation of epithelial cells, which caused implantation failure (Figure 9). These results indicate that the balance of M1 and M2 MФs may be critical for embryonic implantation. Additionally, LIF, essential for implantation, is also known to be produced by $M \Phi$ (29) and $M \Phi$ derived LIF is identified as a potential factor mediating $M \Phi$-epithelial signaling (30). Therefore, the decrease in LIF expression (Figure 3) might be involved in the implantation failure. In our analysis of human samples, the proportion of uterine CD206+ M2-like M $\Phi$, based on the CD206 mRNA expression compared to the total M $\Phi$ marker CD68, was significantly reduced $(P<0.05)$ in nonpregnant patients at the implantation period compared to that in the pregnant patients. And, in non-pregnant patients, the TNF $\alpha$ mRNA expression was significantly increased compared to that in the pregnant patients. Thus, dysregulation of M1/M2 MФs may be one of the causes of implantation failure in humans.
In conclusion, we showed that the depletion of M2 M $\Phi$ led to implantation failure. Further studies are needed to clarify whether the mechanism of implantation failure is due to change in balance of M1/M2 MФs, or decrease in number of M2 MФs.

\section{MATERIALS AND METHODS}

\section{Reagents and Materials}

Roswell Park Memorial Institute (RPMI)-1640 medium and Diphtheria Toxin (DT) were purchased from Sigma-Aldrich (St. Louis, MO, USA). Fetal bovine serum (FBS) was purchased from Life Technologies (Minato-ku Tokyo, Japan). Antibiotics (a mixture of penicillin, streptomycin, and amphotericin B) were purchased from Wako Pure Chemical Industries (Chuo-ku, Osaka, Japan).

\section{Immunohistochemistry}

Paraffin-embedded tissues were cut into $5-\mu \mathrm{m}$-thick sections and mounted on slides. The mouse uterine and ovarian sections were deparaffinized in xylene, rehydrated through a graded series of ethanol, and washed in water. Antigen retrieval was performed in $10 \mathrm{mM}$ sodium citrate buffer $(\mathrm{pH}$ 6.0) in a microwave for $10 \mathrm{~min}$ and then cooling to room temperature. Rabbit IgG was used as a negative control. Slide staining with the first and second antibodies was performed according to the manufacturer's instructions. Immunostaining was performed using antibodies specific to Ki-67 (Abcam, Tokyo, Japan, Cat\# 15580, 1:100 dilution), $\beta$-catenin (Abcam, Tokyo, Japan, Cat\# 138378, 1:100 dilution), phosphorilated-STAT3 (Cell Signaling Technology, Massachusetts, USA, Cat \#9145, 1:100 dilution), and FGF18 (Abcam, Cat\# ab169615, 1:100 dilution). An immunofluorescence analysis of implantation site (uterus) was performed using rabbit anti-mannose receptor (CD206) (Abcam, Cat\# 64693, 1:100 dilution). And the primary antibody was incubated overnight at $4^{\circ} \mathrm{C}$. As a second antibody, the rat anti-rabbit antibody was used. 4',6-diamidino-2-phenylindole (DAPI; 1:500) was used to detect nuclei. Rabbit IgG were used instead of the primary antibody for negative control.

\section{Mice and Diphtheria Toxin Administration}

Female, CD206 DTR mice $(17,18)$, aged 12 to 20 -weeks old were used. The mice were housed in a specific pathogen free (SPF) animal facility with a controlled environment of $22-24^{\circ} \mathrm{C}$ and $60-70 \%$ relative humidity, on a $12 \mathrm{~h}$ light $/ 12 \mathrm{~h}$ dark cycle with food and water provided ad libitum. DT was diluted with sterile phosphate buffered saline (PBS) to the desired concentration and was intra-peritoneally injected to mice to deplete the CD206 positive cells. According to BioGPS, a complete resource for learning about gene and protein function (http://biogps.org/), CD206 seems to be expressed in mouse uterus more than other $\mathrm{M} 2 \mathrm{M} \Phi$ markers. In our preliminary experiment, in each organ, CD206 mRNA seemed to be more expressed than other M2M $\Phi$ markers (data not shown). Decidual MФs are reported to be show higher expression of CD206 (31, 32). Wang et al. (33) reported that the $\mathrm{CD} 206$ expression in $\mathrm{M} \Phi \mathrm{s}$ could be a marker for spontaneous abortion. From these results, CD206 is considered to be a valid marker for uterine M2 M $\Phi$. The experiments 
and procedures were performed at $48 \mathrm{~h}$ after the final DT administration, as previously reported by Nawaz et al. (18). The final DT injection was administered at E 2.5 before implantation. The depletion of CD206 positive cells was confirmed at mRNA levels by qPCR every experiment.

\section{Assessment of the Implantation Site (IS)}

We prepared control group by mating C57/B6 female WT mice with Balb/c male mice, and M2(-) group by mating CD206-DTR female mice with Balb/c mice (Figure 1B). To deplete M2-like $\mathrm{M} \Phi$ s at the implantation period, DT was administered to each mouse at a dose of $30 \mathrm{ng} / \mathrm{gram}$ body weight before implantation. We then checked the implantation sites between two groups. To identify implantation sites on embryonic day 4.5 (E4.5), mice were anesthetized using Avertin (2\% tribromoethanol, $15 \mu \mathrm{l} / \mathrm{g}$ i.p.; Sigma-Aldrich), administered the Chicago blue dye solution ( $0.4 \%$ in PBS i.v.; Sigma-Aldrich) and then analyzed after $10 \mathrm{~min}$. Uteri were dissected and assessed for clearly delineated blue bands as evidence of early implantation sites. In other mice, uterine paraffin sections from control and M2(-) mice were collected on E3.5 and E4.5 and stained with H\&E to assess the implantation sites.

\section{Patients With Implantation Failure}

Uterine endometrial biopsy as performed at the time of the implantation window in 38 patients with implantation failure who visited the outpatient department of obstetrics and gynecology at the University of Tokyo. After the endometrial biopsy, we compared the proportion of uterine M2-like M $\Phi$ to the pan-M $\Phi$ based on the mRNA levels of CD206 and CD68 between the pregnancy and non-pregnant group.

\section{Reverse Transcription (RT) and Quantitative Real-Time Polymerase Chain Reaction (PCR) Analysis}

Total RNA was extracted from the mouse endometrial region from the peritoneal cavity, using the ISOGEN-II (NIPPON GENE, Tokyo, Japan). RT was performed using Rever Tra Ace qPCR RT Master Mix with gDNA Remover (TOYOBO, Tokyo, Japan). About $1.0 \mu \mathrm{g}$ of total RNA was reverse-transcribed in a $20-\mu \mathrm{L}$ volume. For the quantification of various mRNA levels, real-time PCR was performed using the Mx3000P Real-Time PCR System (Agilent Technologies, CA, USA) according to the manufacturer's instructions. The PCR primers used with the SYBR Green protocol were selected from different exons of the corresponding genes to discriminate the PCR products that might arise from possible chromosomal DNA contaminants. The SYBR Green thermal cycling conditions were as follows: 1 cycle of $95^{\circ} \mathrm{C}$ for $30 \mathrm{~s}$, and cycles of $95^{\circ} \mathrm{C}$ for $10 \mathrm{~s}, 60^{\circ} \mathrm{C}$ for $10 \mathrm{~s}$ and $72^{\circ} \mathrm{C}$ for $10 \mathrm{~s}$. The primer sequences used were as follows: 3-phosphate dehydrogenase (GAPDH, NM_002046: 628-648 and 1079-1060), mouse CD206 (NM_000710.3: 326-347 and 495-473), mouse TNFo (NM_000623.3: 432-453 and 605-584), mouse IL-10 (NM_010548.2: 390-412 and 464443), mouse CD11c (NM_001363985.1: 82-103 and 194-175), mouse iNOS (NM_001313922.1: 2363-2382 and 2489-2470), $\beta$-catenin (NM_000623.3: 432-453 and 605-584), Fibroblast
Growth Factor 18 (NM_000623.3: 432-453 and 605-584), Wnt 4 (NM_009523.2: 318-337 and 426-409), Wnt 5A (NM_009524.4: 565-583 and 668-650), Wnt 6 (XM_006495889.2: 670-688 and 796-779), Wnt 7A (NM_001363757.1: 501-518 and 578558), and Wnt 7B (NM_009528.3: 421-440 and 492-472). The relative mRNA levels were calculated using the standard curve method and were normalized to the mRNA levels of GAPDH (forward, 5'-AATGTGTCCGTCGTGGATCTGA$3^{\prime}$ and reverse, 5'-GATGCCTGCTTCACCACCTTCT-3') (Supplementary Data 1).

\section{Measurement of Estradiol (E2) and Progesterone (P4) Levels}

Mouse blood samples were collected during the analysis. Plasma levels of E2 and P4 were measured in duplicate using the specific EIA kits (Cayman, USA).

\section{Statistical Analysis}

Data were evaluated by Mann Whitney test using Jump version 10. $P<0.05$ was accepted as statistically significant.

\section{DATA AVAILABILITY STATEMENT}

The datasets generated for this study can be found in online repositories. The names of the repository/repositories and accession number(s) can be found in the article/Supplementary Material.

\section{ETHICS STATEMENT}

The studies involving human participants were reviewed and approved by the committee of the University of Tokyo (10991). The patients/participants provided their written informed consent to participate in this study. The animal study was reviewed and approved by the committee of University of Toyama (A 2015med-55).

\section{AUTHOR CONTRIBUTIONS}

SS, OY, KT, YH, and YOn: conception and design. YOn, ANak and $\mathrm{AU}$ : acquiring and processing samples. YOn, ANaw, YF, AU, OY, and ES: execution of the experiment. YOn and OY: analysis of data. SS, OY, ANaw, TH, ANak, and YOs: interpretation of data. YOn and OY: drafting the manuscript. SS, OY, YOn, and SW: revision of the manuscript for important intellectual content. All authors contributed to the article and approved the submitted version.

\section{FUNDING}

This work was supported by grants from Ministry of Education, Culture, Sports, Science and Technology in Japan [KAKENHI Grant Number 15H04980 (SS)] and AMED under Grant Number JP17gk0110018 (SS) and the Smoking Research Foundation. 


\section{ACKNOWLEDGMENTS}

The authors thank Mina Matsuo, Kaori Nomoto, and Hideki Hatta for their technical support and thank Editage (www.editage.com) for English language review.

\section{REFERENCES}

1. Gordon S. The macrophage: past, present and future. Eur J Immunol. (2007) 37:S9-17. doi: 10.1002/eji.200737638

2. Wynn TA, Chawla A, Pollard JW. Origins and hallmarks of macrophages: development, homeostasis, and disease. Nature. (2013) 496:445-55. doi: 10.1038/nature12034

3. Kebin H, Yang J, Zissis C, Xiaodong H, Hao L, Ling L. Macrophage functions and regulation: roles in diseases and implications in therapeutics. J Immunol Res. (2018) 2018:7590350. doi: 10.1155/2018/7590350

4. Yan CL, Xian BZ, Yan FC, Yong MY. Macrophage polarization in inflammatory diseases. Int J Biol Sci. (2014) 10:520-9. doi: 10.7150/ ijbs. 8879

5. Zhang L. Contribution of resident and recruited macrophages in vascular physiology and -athology. Curr Opin Hematol. (2018) 25:196-203. doi: 10.1097/MOH.0000000000000421

6. Schultze JL, Schmieder A, Goerdt S. Macrophage activation in human diseases. Semin Immunol. (2015) 27:249-56. doi: 10.1016/j.smim.2015. 07.003

7. Decano JL, Aikawa M. Dynamic macrophages: understanding mechanisms of activation as guide to therapy for atherosclerotic vascular disease. Front Cardiovasc Med. (2018) 5:97. doi: 10.3389/fcvm.2018.00097

8. Egashira M, Hirota Y. Uterine receptivity and embryo-uterine interactions in embryo implantation: lessons from mice. Reprod Med Biol. (2013) 12:127-32. doi: 10.1007/s12522-013-0153-1

9. Cha J, Sun X, Dey SK. Mechanisms of implantation: strategies for successful pregnancy. Nat Med. (2012) 18:1754-67. doi: 10.1038/nm.3012

10. Dey SK, Lim H, Das SK, Reese J, Paria BC, Daikoku T, et al. Molecular cues to implantation. Endocr Rev. (2004) 25:341-73. doi: 10.1210/er.20030020

11. Bilinski P, Roopenian D, Gossler A. Maternal IL-11r alpha function is required for normal decidua and fetoplacental development in mice. Genes Dev. (1998) 12:2234-43. doi: 10.1101/gad.12.14.2234

12. Daikoku T, Cha J, Sun X, Tranguch S, Xie H, Fujita T, et al. Conditional deletion of Msx homeobox genes in the uterus inhibits blastocyst implantation by altering uterine receptivity. Dev Cell. (2011) 21:1014-25. doi: 10.1016/j.devcel.2011.09.010

13. Sun X, Bartos A, Whitsett JA, Dey SK. Uterine deletion of Gp130 or Stat3 shows implantation failure with increased estrogenic responses. $\mathrm{Mol}$ Endocrinol. (2013) 27:1492-501. doi: 10.1210/me.2013-1086

14. Care AS, Diener KR, Jasper MJ, Brown HM, Ingman WV, Robertson SA. Macrophages regulate corpus luteum development during embryo implantation in mice. J Clin Invest. (2013) 123:3472-87. doi: 10.1172/JCI60561

15. Gordon S, Martinez FO. Alternative activation of macrophages: mechanism and functions. Immunity. (2010) 32:593-604. doi: 10.1016/j.immuni.2010.05.007

16. Martinez FO, Gordon S. The M1 and M2 paradigm of macrophage activation: time for reassessment. F1000Prime Rep. (2014) 6:13. doi: 10.12703/P6-13

17. Kambara K, Ohashi W, Tomita K, Takashina M, Fujisaka S, Hayashi $\mathrm{R}$, et al. In vivo depletion of CD206+ M2 macrophages exaggerates lung injury in endotoxemic mice. Am J Pathol. (2015) 185:162-71. doi: 10.1016/j.ajpath.2014.09.005

18. Nawaz A, Aminuddin A, Kado T, Takikawa A, Yamamoto S, Tsuneyama K, et al. CD206+ M2-like macrophages regulate systemic glucose metabolism by inhibiting proliferation of adipocyte progenitors. Nat Commun. (2017) 8:286. doi: 10.1038/s41467-017-00231-1

\section{SUPPLEMENTARY MATERIAL}

The Supplementary Material for this article can be found online at: https://www.frontiersin.org/articles/10.3389/fimmu. 2020.557184/full\#supplementary-material

Supplementary Data 1 | Primer sequences of mouse and human.

19. Ono Y, Nagai M, Yoshino O, Koga K, Nawaz A, Hatta H, et al. CD11c+ M1-like macrophages (MФs) but not CD206+ M2-like MФs are involved in folliculogenesis in mice ovary. Sci Rep. (2018) 8:8171. doi: 10.1038/s41598-018-25837-3

20. Douglas NC, Zimmermann RC, Tan QK, Sullivan-Pyke CS, Sauer MV, Kitajewski JK, et al. VEGFR-1 blockade disrupts peri-implantation decidual angiogenesis and macrophage recruitment. Vasc Cell. (2014) 6:16. doi: 10.1186/2045-824X-6-16

21. Stewart CL, Kaspar P, Brunet LJ, Bhatt H, Gadi I, Köntgen F, et al. Blastocyst implantation depends on maternal expression of leukaemia inhibitory factor. Nature. (1992) 359:76-9. doi: 10.1038/359 $076 \mathrm{a} 0$

22. Mohamed OA, Jonnaert M, Labelle-Dumais C, Kuroda K, Clarke HJ Dufort D. Uterine Wnt/beta-catenin signaling is required for implantation. Proc Natl Acad Sci USA. (2005) 102:8579-84. doi: 10.1073/pnas.05006 12102

23. Oguma K, Oshima $H$, Aoki $M$, Uchio R, Naka K, Nakamura S, et al. Activated macrophages promote Wnt signalling through tumour necrosis factor-a in gastric tumour cells. EMBO J. (2008) 27:1671-81. doi: 10.1038/emboj.2008.105

24. Care AS, Ingman WV, Moldenhauer LM, Jasper MJ, Robertson SA. Ovarian steroid hormone-regulated uterine remodeling occurs independently of macrophages in mice. Biol Reprod. (2014) 91:60. doi: 10.1095/biolreprod.113.116509

25. Plaks V, Birnberg T, Berkutzki T, Sela S, BenYashar A, Kalchenko V, et al. Uterine DCs are crucial for decidua formation during embryo implantation in mice. J Clin Invest. (2008) 118:3954-65. doi: 10.1172/JCI 36682

26. Hirota Y. Progesterone governs endometrial proliferation differentiation switching and blastocyst implantation. Endocr J. (2019) 66:199-206. doi: 10.1507/endocrj.EJ18-0431

27. Haraguchi H, Saito-Fujita T, Hirota Y, Egashira M, Matsumoto L, Matsuo M, et al. MicroRNA-200a locally attenuates progesterone signaling in the cervix, preventing embryo implantation. Mol Endocrinol. (2014) 28:1108-17. doi: 10.1210/me.2014-1097

28. Nallasamy S, Li Q, Bagchi MK, Bagchi IC. Msx homeobox genes critically regulate embryo implantation by controlling paracrine signaling between uterine stroma and epithelium. PLoS Genet. (2012) 8:e1002500. doi: 10.1371/journal.pgen.1002500

29. Nakamura H, Jasper MJ, Hull ML, Apilin JD, Robertson SA. Macrophages regulate expression of $\alpha 1,2$-fucosyltransferase genes in human endometrial epithelial cells. Mol Hum Reprod. (2011) 18:204-15. doi: 10.1093/molehr/gar070

30. Jasper MJ, Care AS, Sullivan B, Ingman WV, Aplin JD, Robertson SA. Macrophage-derived LIF and IL1B regulate alpha $(1,2)$ fucosyltransferase 2 (Fut2) expression in mouse uterine epithelial cells during early pregnancy. Biol Reprod. (2011) 84:179-88. doi: 10.1095/biolreprod.110. 085399

31. Laskarin G, Cupurdija K, Tokmadzic VS, Dorcic D, Dupor J, Juretic $\mathrm{K}$, et al. The presence of functional mannose receptor on macrophages at the maternal-fetal interface. Hum Reprod. (2005) 20:1057-66. doi: 10.1093/humrep/deh740

32. Svensson J, Jenmalm MC, Matussek A, Geffers R, Berg G, Ernerudh J. Macrophages at the fetal-maternal interface express markers of alternative activation and are induced by M-CSF and IL-10. J Immunol. (2011) 187:367182. doi: 10.4049/jimmunol.1100130 
33. Wang WJ, Hao CF, Lin QD. Dysregulation of macrophage activation by decidual regulatory $\mathrm{T}$ cells in unexplained recurrent miscarriage patient. $J$ Reprod Immunol. (2011) 92:97-102. doi: 10.1016/j.jri.2011.08.004

Conflict of Interest: The authors declare that the research was conducted in the absence of any commercial or financial relationships that could be construed as a potential conflict of interest.
Copyright (C) 2020 Ono, Yoshino, Hiraoka, Sato, Fukui, Ushijima, Nawaz, Hirota Wada, Tobe, Nakashima, Osuga and Saito. This is an open-access article distributed under the terms of the Creative Commons Attribution License (CC BY). The use, distribution or reproduction in other forums is permitted, provided the original author(s) and the copyright owner(s) are credited and that the original publication in this journal is cited, in accordance with accepted academic practice. No use, distribution or reproduction is permitted which does not comply with these terms. 